\title{
EXTENSIVE PHOTOMETRY OF V1838 AQL DURING THE 2013 SUPEROUTBURST
}

\author{
J. Echevarría ${ }^{1}$, E. de Miguel ${ }^{2}$, J. V. Hernández Santisteban ${ }^{3}$, R. Michel ${ }^{4}$, R. Costero ${ }^{1}$, L. J. Sánchez ${ }^{1}$, \\ A. Ruelas-Mayorga ${ }^{1}$, J. Olivares ${ }^{5}$, D. González-Buitrago ${ }^{6}$, J. L. Jones ${ }^{7}$, A. Oskanen ${ }^{8}$, W. Goff ${ }^{9}$, J. Ulowetz $^{10}$, \\ G. Bolt ${ }^{11}$, R. Sabo ${ }^{12}$, F.-J Hambsch ${ }^{13}$, D. Slauson ${ }^{14}$, and W. Stein ${ }^{15}$ \\ Received August 8 2018; accepted October 242018
}

\begin{abstract}
We present an in-depth photometric study of the 2013 superoutburst of the recently discovered cataclysmic variable V1838 Aql and subsequent photometry near its quiescent state. A careful examination of the development of the superhumps is presented. Our best determination of the orbital period is $P_{\text {orb }}=0.05698(9)$ days, based on the periodicity of early superhumps. Comparing the superhump periods at stages $A$ and $B$ with the early superhump value we derive a period excess of $\epsilon=0.024(2)$ and a mass ratio of $q=0.10(1)$. We suggest that V1838 Aql is approaching the orbital period minimum and thus has a low-mass star as a donor instead of a substellar object.
\end{abstract}

\section{RESUMEN}

Presentamos un estudio fotométrico detallado de la super-erupción de V1838 Aql, una variable cataclísmica recientemente descubierta, desde el máximo en 2013 hasta su regreso al mínimo. Examinamos en detalle la evolución de las superjorobas (superhumps). Determinamos el período orbital $P_{\text {orb }}=0.05698(9)$ días a partir de la periodicidad de las superjorobas tempranas. Comparando los períodos de las superjorobas en las etapas $A$ y $B$ con el valor del período orbital, derivamos un valor del cambio en el período orbital de $\epsilon=0.024(2)$ y un cociente de masa para el sistema de $q=0.10(1)$. Sugerimos que V1838 Aql se está acercando al mínimo período orbital, por lo que la secundaria sería una estrella de baja masa y no un objeto sub-estelar.

Key Words: techniques: photometric — stars: novae, cataclysmic variables — stars: dwarf novae - stars: individual: V1838 Aql

\footnotetext{
${ }^{1}$ Instituto de Astronomía, Universidad Nacional Autónoma de México, México.

${ }^{2}$ Departamento de Ciencias Integradas, Universidad de Huelva, Huelva, Spain.

${ }^{3}$ Anton Pannekoek Institute for Astronomy, University of Amsterdam, Amsterdam, The Netherlands.

${ }^{4}$ Instituto de Astronomía, Universidad Nacional Autónoma de México, Ensenada, Baja California, México.

${ }^{5}$ Laboratoire d'astrophysique de Bordeaux, Univ. Bordeaux, Pessac, France.

${ }^{6}$ Departament of Physics and Astronomy, University of California, Irvine, CA, USA.

${ }^{7}$ CBA-Oregon, Jack Jones Observatory, Aurora, OR, USA.

${ }^{8}$ CBA-Finland, Hankasalmi Observatory, Muurame, Finland.

${ }^{9}$ CBA-California, Sutter Creek, CA, USA.

${ }^{10}$ CBA-Illinois, Northbrook Meadow Observatory, Northbrook, IL, USA.

${ }^{11}$ CBA-Australia, Craigie, Western Australia, Australia.

${ }^{12}$ CBA-Montana, Bozeman, MT, USA.

${ }^{13} \mathrm{CBA}-\mathrm{Mol}$, Andromeda Observatory, Mol, Belgium.
}

\section{INTRODUCTION}

Cataclysmic variables (CVs) are close binary systems in which a white dwarf (WD) accretes from a low-mass star via Roche-lobe overflow, often creating an accretion disc (for a review see Warner 1995). A large fraction of CVs belong to the subclass of dwarf novae (DNe). They undergo recurrent outbursts with typical amplitudes of $\approx 2-6 \mathrm{mag}$ in the optical, which are commonly accepted to be caused by a thermal-viscous instability in the disc (Osaki 1974). In addition, SU UMa-type DNe (the subclass of DNe systems with short orbital periods, $P_{\text {orb }}<2.5 \mathrm{hr}$ ) exhibit occasional eruptions that are less frequent, longer lasting, and slightly brighter (by $\approx 0.5-1.0 \mathrm{mag}$ ) than the normal out-

\footnotetext{
${ }^{14}$ CBA-Iowa, Owl Ridge Observatory, IA, USA.

${ }^{15}$ CBA-Las Cruces, Las Cruces, NM, USA.
} 
bursts. The key feature during these so-called superoutbursts is the presence of superhumps a modulation in the light curve with an underlying periodicity, $P_{\mathrm{sh}}$, a few percent longer than the orbital period. They are thought to arise from a precessing non-axisymmetric disc (Vogt 1982), with the eccentricity being produced by the tidal instability developed at the radius of the 3:1 resonance (Whitehurst 1988). The analysis of the timing and evolution of such light oscillations provides estimates of the system's parameters using empirical (Patterson et al. 2005) and theoretical (Kato \& Osaki 2013) relationships between the superhump/orbital period excess, $\epsilon \equiv\left(P_{\text {sh }}-P_{\text {orb }}\right) / P_{\text {orb }}$, and the binary mass ratio $q \equiv M_{2} / M_{1}$. Several $\epsilon(q)$ relations have been proposed by these authors based on different stages of the superhumps, although Otulakowska-Hypka et al. (2016) show that the scatter in the $\epsilon-q$ diagram is considerable for very short orbital periods. This is due to a lack of objects with dynamically confirmed small values of $q$ (Patterson 2011; Kato \& Osaki 2013).

Among the SU UMa-type systems, there is a large group that accumulates around the minimum of the orbital period distribution of CVs $\left(P_{\text {orb }} \approx 78 \mathrm{~min}\right)$ (Paczynski \& Sienkiewicz 1981; Gänsicke et al. 2009; Knigge et al. 2011). These are binaries with extremely low mass-transfer rates, named WZ Sge-type objects, which are characterised by rare (commonly detected every $\approx 10$ years), and large amplitude superoutbursts of duration of $\approx 30$ days, caused by an instability in low viscosity accretion discs with $\alpha \approx 0.01-0.001$ (Smak 1993; Osaki 1994). Some of them are systems currently evolving towards longer periods and are collectively known as period bouncers (e.g. Littlefair, Dhillon \& Martin 2003). These binaries are expected to harbour a substellar secondary companion (Howell et al. 1997), i.e. brown dwarfs (e.g. Littlefair et al. 2006; Harrison 2016; Hernández Santisteban et al. 2016; Neustroev et al. 2017).

Worth noting is that WZ Sge-type objects are characterised not only by a long superoutburst recurrence time in comparison to typical SU UMa stars, but also by the presence of early superhumps (double-wave modulation) during the first few days of the eruption, with a periodicity $\left(P_{\text {esh }}\right)$ essentially equal to the orbital period of the binary (details in O'Donoghue et al. 1991; Kato 2015). The advent of all-sky surveys (e.g. Breedt et al. 2014) and worldwide citizen-telescope networks has contributed to the discovery of a large population of faint DNe. Among these discoveries, the elusive population of short-period systems, in particular period bouncers, has been found and investigated (Patterson 2011; Coppejans et al. 2016; Otulakowska-Hypka et al. 2016).

The discovery of a new transient, initially proposed as possible nova, was reported by Itagaki on 2013 May 31. Henden ${ }^{16}$ pointed out that the colour indices of the object and the un-reddened field suggested a DN rather than a nova. As pointed out by Hurst ${ }^{17}$, Kojima reported a pre-discovery image on 2013 May 30.721 UT, when the magnitude was at about $9.8 \mathrm{mag}$ (un-filtered). A CBET report of this new DNe in Aquila, can be found in Itagaki (2013).

Although a preliminary analysis of the behaviour of V1838 Aql (originally designated as PNV J19150199+0719471) was published by Kato et al. (2014), we present here a full analysis of the superhump behaviour based on our extensive data.

In $\S 2$ we present the observations and their reduction methods. The photometric data and the period analysis are presented in $\S 3$, while in $\S 4$ we address the discussion of our results. We present our conclusions in $\S 5$.

\section{OBSERVATIONS AND REDUCTION}

Photometric observations in the $V$ band were obtained in 2013 during the nights of June 3, 4, $5,6,17,18,28$ and September 2 and 25 at the $0.84 \mathrm{~m}$ telescope of the Observatorio Astronómico Nacional at San Pedro Mártir (SPM). We used the Blue-ESOPO CCD detector ${ }^{18}$ on a $2 \times 2$ binning configuration. The exposure time of the SPM observations varied between 10 and $30 \mathrm{~s}$. In addition, time-series photometry of the superoutburst was obtained from 10 observatories of the Center for Backyard Astrophysics (CBA) -a network of small $(0.2-0.4 \mathrm{~m})$ telescopes that covers a wide range in terrestrial longitude. Skillman \& Patterson (1993) and de Miguel et al. (2016) describe the methods and observing stations of the CBA network. These observations amounted to 162 separate time-series during 58 nights from June 1 to August 2, 2013, and the typical exposure time ranged from 20 to 120 seconds, depending on the brightness of V1838 Aql. Nearly half of these observations were obtained in $V$ light, while the rest (mainly during the post-outburst regime) were unfiltered. We did not attempt any absolute calibration of the data during the eruption, but the magnitude scale is expected to resemble

\footnotetext{
${ }^{16}$ http://ooruri.kusastro.kyoto-u.ac.jp/mailarchive/ vsnet-alert/15772).

17 www . theastronomer.org/tacirc/2013/e2919.txt.

${ }^{18} \mathrm{http}$ ://www. astrossp.unam.mx/indexspm.html.
} 
closely $V$ magnitudes with a zero-point uncertainty of $\approx 0.05$ mag. Further observations in the $R$ band using the $2.1 \mathrm{~m}$ telescope at SPM during 2018, July 18 were conducted. Unfortunately, the weather was unstable and we only managed to obtain differential photometry over three orbital cycles. In the following, we report times and refer to specific dates in a truncated form defined as HJD - 2, 456, 000 .

\section{PHOTOMETRY AND PERIOD ANALYSIS}

\subsection{Photometric Observations}

Most of our photometric observations come from the CBA network, with additional $V$-band observations obtained with the $0.84 \mathrm{~m}$ telescope at SPM at some critical stages of the outburst and during the late decline. ${ }^{19}$ We observed the typical pattern seen in SU UMa stars during superoutburst: a plateau phase -lasting $\approx 25$ days, from HJD 444 to 469 - where the mean brightness varied smoothly from 10.5 to $13.0 \mathrm{mag}$, followed by a rapid decline ( $\approx 3$ mag in 2 days) at the end of the main eruption. The subsequent fading towards quiescence occurred at a rather low rate $\left(\approx 0.035 \mathrm{mag} \mathrm{d}^{-1}\right)$, and even 3.5 years after the end of the eruption, the system was found to be $\approx 0.5 \mathrm{mag}$ above the pre-eruption quiescent brightness. However, based on the observations obtained with the NTT telescope (La Palma, Spain), we confirmed that the object had reached the pre-outburst level by June 2017. These observations and the general spectral distribution at quiescence have no further relevance here and will be discussed in a future publication.

\subsection{Full Analysis of the Different Stages of the Superhump}

Our primary tool for studying periodic signals was the Period 04 package (Lenz \& Breger 2005). First, we subtracted the mean and (linear) trend from each individual light curve and formed nightlyspliced light curves. Then, after combining light curves from adjacent nights, a search for periodic signals was done. This approach allows us to improve the frequency resolution, but it has to be implemented with caution, since variations in the amplitude and/or the period of the modulation -both effects known to afflict erupting $\mathrm{DNe}-$ can distort the outcome of the frequency analysis.

A general view of the superhump transitions can be looked up by identifying the different stages of the

${ }^{19}$ All CBA and SPM data are available on request.

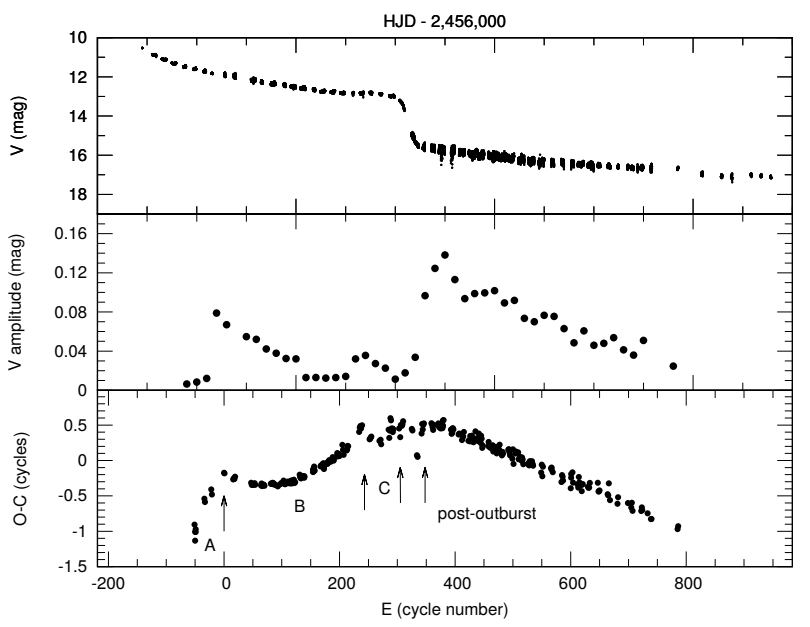

Fig. 1. Photometric behaviour of V1838 Aql during its superoutburst in 2013. Top frame: global light curve of the observational campaign. Middle frame: amplitude variations of the superhumps along the superoutburst (see text). Bottom frame: $\mathrm{O}-\mathrm{C}$ diagram for the superhump maxima in the HJD 449.6-498.6 day interval with respect to the ephemeris given in equation (1). The arrows indicate the (approximate) location of transitions between different regimes of superhump period variations (see text).

superhumps: early superhumps, Stages $A, B$ and $C$ as well as the post-outburst stage (see Kato et al. 2009,2014 , for this terminology in our general discussion). Thus, we looked at the time variations of the superhump period and its amplitude by examining the variation in time with respect to a welldefined feature of the superhump signal.

After this general analysis, a detailed explanation of these stages was made. First, we derived the timings of superhump maxima. A total of 310 times of superhump maxima was identified in the light curves in the interval HJD 449.6-498.6 days. These maxima are shown in Table 1. The early stage of the eruption was not considered, since the signal there was of very low amplitude and individual maxima were not well defined. A linear regression to these timings provides the following test ephemeris:

$$
T_{\max }(\mathrm{HJD})=2,456,452.8035(23)+0.058191(6) E .
$$

The top panel of Figure 1 displays the general photometric behaviour of the system during our campaign. Next, on the middle panel of Figure 1 is presented the variation of the amplitude of the superhump modulation, defined as the semi-amplitude of the sine wave that best fits the nightly photometric data. 
TABLE 1

TIMES OF SUPERHUMP MAXIMA OF V1838 Aql DURING THE 2013 SUPEROUTBURST. ${ }^{a}$

\begin{tabular}{|c|c|c|c|c|c|c|c|c|c|c|c|}
\hline$E^{b}$ & $T_{-} \max ^{\mathrm{c}}$ & $\mathrm{O}-\mathrm{C}^{\mathrm{d}}$ & $\mathrm{E}^{\mathrm{b}}$ & $T_{\text {_max }}{ }^{\mathrm{c}}$ & $\mathrm{O}-\mathrm{C}^{\mathrm{d}}$ & $\mathrm{E}^{\mathrm{b}}$ & $T_{\text {_max }}{ }^{\mathrm{c}}$ & $\mathrm{O}-\mathrm{C}^{\mathrm{d}}$ & $\mathrm{E}^{\mathrm{b}}$ & $T_{-} \max ^{\mathrm{c}}$ & $\mathrm{O}-\mathrm{C}^{\mathrm{d}}$ \\
\hline-51 & 449.7831 & -0.906 & 116 & 459.5372 & -0.283 & 189 & 463.8032 & 0.028 & 345 & 472.9088 & 0.506 \\
\hline-50 & 449.8282 & -1.131 & 116 & 459.5365 & -0.295 & 190 & 463.8598 & -0.001 & 346 & 472.9681 & 0.524 \\
\hline-50 & 449.8354 & -1.007 & 117 & 459.5944 & -0.301 & 190 & 463.8607 & 0.016 & 359 & 473.7247 & 0.527 \\
\hline-49 & 449.8934 & -1.011 & 117 & 459.5939 & -0.309 & 195 & 464.1540 & 0.056 & 360 & 473.7820 & 0.511 \\
\hline-49 & 449.8956 & -0.972 & 118 & 459.6513 & -0.322 & 196 & 464.2150 & 0.104 & 361 & 473.8381 & 0.474 \\
\hline-34 & 450.7936 & -0.541 & 120 & 459.7703 & -0.278 & 197 & 464.2728 & 0.097 & 362 & 473.8938 & 0.431 \\
\hline-33 & 450.8491 & -0.586 & 121 & 459.8289 & -0.271 & 198 & 464.3288 & 0.060 & 363 & 473.9561 & 0.502 \\
\hline-22 & 451.4995 & -0.410 & 122 & 459.8842 & -0.320 & 199 & 464.3889 & 0.092 & 371 & 474.4228 & 0.523 \\
\hline-21 & 451.5535 & -0.481 & 123 & 459.9432 & -0.307 & 200 & 464.4455 & 0.065 & 372 & 474.4782 & 0.475 \\
\hline 0 & 452.7934 & -0.174 & 131 & 460.4149 & -0.200 & 205 & 464.7456 & 0.222 & 373 & 474.5387 & 0.514 \\
\hline 1 & 452.8512 & -0.181 & 132 & 460.4708 & -0.239 & 206 & 464.7997 & 0.151 & 374 & 474.5940 & 0.464 \\
\hline 16 & 453.7190 & -0.268 & 132 & 460.4718 & -0.223 & 207 & 464.8537 & 0.080 & 375 & 474.6515 & 0.453 \\
\hline 17 & 453.7782 & -0.250 & 133 & 460.5281 & -0.254 & 212 & 465.1482 & 0.141 & 376 & 474.7119 & 0.490 \\
\hline 18 & 453.8358 & -0.261 & 133 & 460.5286 & -0.247 & 213 & 465.2072 & 0.154 & 377 & 474.7702 & 0.492 \\
\hline 19 & 453.8959 & -0.228 & 134 & 460.5883 & -0.220 & 214 & 465.2684 & 0.205 & 377 & 474.7705 & 0.497 \\
\hline 47 & 455.5203 & -0.313 & 134 & 460.5883 & -0.220 & 215 & 465.3263 & 0.201 & 377 & 474.7706 & 0.500 \\
\hline 48 & 455.5766 & -0.345 & 135 & 460.6459 & -0.231 & 234 & 466.4437 & 0.403 & 378 & 474.8263 & 0.456 \\
\hline 49 & 455.6356 & -0.331 & 136 & 460.7049 & -0.218 & 235 & 466.5055 & 0.465 & 378 & 474.8280 & 0.486 \\
\hline 50 & 455.6938 & -0.331 & 137 & 460.7625 & -0.227 & 236 & 466.5650 & 0.488 & 379 & 474.8868 & 0.497 \\
\hline 51 & 455.7512 & -0.345 & 138 & 460.8201 & -0.237 & 237 & 466.6212 & 0.453 & 379 & 474.8862 & 0.486 \\
\hline 52 & 455.8099 & -0.336 & 139 & 460.8780 & -0.243 & 239 & 466.7401 & 0.497 & 379 & 474.8909 & 0.566 \\
\hline 53 & 455.8690 & -0.321 & 139 & 460.8787 & -0.230 & 252 & 467.4850 & 0.298 & 380 & 474.9493 & 0.570 \\
\hline 54 & 455.9259 & -0.342 & 139 & 460.8792 & -0.221 & 253 & 467.5438 & 0.309 & 393 & 475.6979 & 0.435 \\
\hline 64 & 456.5073 & -0.351 & 154 & 461.7554 & -0.164 & 254 & 467.6029 & 0.324 & 394 & 475.7561 & 0.435 \\
\hline 65 & 456.5671 & -0.323 & 154 & 461.7576 & -0.126 & 255 & 467.6619 & 0.338 & 394 & 475.7527 & 0.376 \\
\hline 66 & 456.6232 & -0.361 & 155 & 461.8140 & -0.158 & 269 & 468.4730 & 0.277 & 395 & 475.8151 & 0.449 \\
\hline 69 & 456.7989 & -0.341 & 156 & 461.8764 & -0.085 & 271 & 468.5903 & 0.292 & 395 & 475.8118 & 0.392 \\
\hline 70 & 456.8561 & -0.358 & 156 & 461.8756 & -0.098 & 272 & 468.6448 & 0.229 & 395 & 475.8140 & 0.430 \\
\hline 81 & 457.4979 & -0.328 & 157 & 461.9311 & -0.144 & 285 & 469.4134 & 0.436 & 396 & 475.8725 & 0.436 \\
\hline 82 & 457.5560 & -0.330 & 167 & 462.5166 & -0.083 & 286 & 469.4648 & 0.320 & 396 & 475.8737 & 0.456 \\
\hline 83 & 457.6146 & -0.323 & 168 & 462.5755 & -0.070 & 287 & 469.5288 & 0.420 & 397 & 475.9309 & 0.439 \\
\hline 84 & 457.6733 & -0.314 & 169 & 462.6334 & -0.076 & 288 & 469.5973 & 0.598 & 406 & 476.4504 & 0.366 \\
\hline 85 & 457.7303 & -0.335 & 170 & 462.6916 & -0.075 & 289 & 469.6537 & 0.567 & 407 & 476.5093 & 0.380 \\
\hline 86 & 457.7893 & -0.320 & 171 & 462.7489 & -0.090 & 290 & 469.7051 & 0.451 & 411 & 476.7418 & 0.374 \\
\hline 87 & 457.8469 & -0.332 & 171 & 462.7500 & -0.072 & 291 & 469.7620 & 0.428 & 412 & 476.7950 & 0.288 \\
\hline 88 & 457.9044 & -0.343 & 171 & 462.7514 & -0.047 & 292 & 469.8186 & 0.400 & 413 & 476.8565 & 0.345 \\
\hline 92 & 458.1365 & -0.354 & 172 & 462.8101 & -0.039 & 292 & 469.8215 & 0.450 & 414 & 476.9143 & 0.338 \\
\hline 93 & 458.1941 & -0.365 & 172 & 462.8074 & -0.086 & 293 & 469.8789 & 0.437 & 415 & 476.9763 & 0.404 \\
\hline 97 & 458.4284 & -0.338 & 172 & 462.8078 & -0.078 & 303 & 470.4617 & 0.452 & 423 & 477.4346 & 0.280 \\
\hline 99 & 458.5459 & -0.318 & 173 & 462.8644 & -0.106 & 305 & 470.5709 & 0.329 & 425 & 477.5504 & 0.270 \\
\hline 102 & 458.7208 & -0.313 & 173 & 462.8635 & -0.122 & 306 & 470.6391 & 0.501 & 426 & 477.6130 & 0.346 \\
\hline 103 & 458.7807 & -0.283 & 178 & 463.1596 & -0.033 & 308 & 470.7548 & 0.489 & 427 & 477.6698 & 0.321 \\
\hline 104 & 458.8372 & -0.313 & 179 & 463.2169 & -0.049 & 309 & 470.8143 & 0.510 & 429 & 477.7832 & 0.270 \\
\hline 105 & 458.8942 & -0.333 & 180 & 463.2767 & -0.021 & 310 & 470.8750 & 0.554 & 429 & 477.7877 & 0.348 \\
\hline 106 & 458.9522 & -0.336 & 181 & 463.3344 & -0.030 & 325 & 471.7415 & 0.446 & 430 & 477.8417 & 0.275 \\
\hline 110 & 459.1872 & -0.298 & 182 & 463.3946 & 0.005 & 326 & 471.7985 & 0.424 & 430 & 477.8434 & 0.305 \\
\hline 111 & 459.2441 & -0.321 & 184 & 463.5105 & -0.003 & 334 & 472.2435 & 0.072 & 431 & 477.9022 & 0.315 \\
\hline 112 & 459.3025 & -0.316 & 185 & 463.5680 & -0.015 & 335 & 472.3005 & 0.051 & 432 & 477.9570 & 0.257 \\
\hline 113 & 459.3617 & -0.300 & 186 & 463.6273 & 0.003 & 342 & 472.7271 & 0.381 & 435 & 478.1406 & 0.412 \\
\hline 115 & 459.4778 & -0.305 & 187 & 463.6863 & 0.019 & 343 & 472.7931 & 0.516 & 436 & 478.1927 & 0.308 \\
\hline 115 & 459.4767 & -0.323 & 188 & 463.7462 & 0.047 & 344 & 472.8467 & 0.437 & 437 & 478.2500 & 0.293 \\
\hline
\end{tabular}


TABLE 1. CONTINUED

\begin{tabular}{|c|c|c|c|c|c|c|c|c|c|c|c|}
\hline$E^{b}$ & $\mathrm{~T} \max ^{\mathrm{c}}$ & $\mathrm{O}-\mathrm{C}^{\mathrm{d}}$ & $\mathrm{E}^{\mathrm{b}}$ & $\mathrm{T}_{-} \max ^{\mathrm{c}}$ & $\mathrm{O}-\mathrm{C}^{\mathrm{d}}$ & $E^{b}$ & $\mathrm{~T}_{-} \max ^{\mathrm{c}}$ & $\mathrm{O}-\mathrm{C}^{\mathrm{d}}$ & $E^{b}$ & $\mathrm{~T}_{-} \max ^{\mathrm{c}}$ & $\mathrm{O}-\mathrm{C}^{\mathrm{d}}$ \\
\hline 438 & 478.3092 & 0.310 & 482 & 480.8586 & 0.120 & 535 & 483.9315 & -0.073 & 631 & 489.4996 & -0.386 \\
\hline 440 & 478.4235 & 0.273 & 483 & 480.9154 & 0.097 & 549 & 484.7405 & -0.170 & 632 & 489.5580 & -0.382 \\
\hline 441 & 478.4880 & 0.381 & 492 & 481.4423 & 0.151 & 550 & 484.8051 & -0.060 & 633 & 489.6199 & -0.318 \\
\hline 442 & 478.5401 & 0.277 & 493 & 481.5009 & 0.159 & 551 & 484.8538 & -0.222 & 647 & 490.4349 & -0.313 \\
\hline 443 & 478.6004 & 0.313 & 494 & 481.5564 & 0.112 & 552 & 484.9199 & -0.087 & 648 & 490.4859 & -0.437 \\
\hline 444 & 478.6549 & 0.251 & 495 & 481.6122 & 0.071 & 566 & 485.7333 & -0.109 & 649 & 490.5440 & -0.438 \\
\hline 445 & 478.7189 & 0.351 & 499 & 481.8423 & 0.026 & 567 & 485.7904 & -0.128 & 650 & 490.6030 & -0.424 \\
\hline 446 & 478.7747 & 0.309 & 500 & 481.9082 & 0.158 & 567 & 485.7921 & -0.098 & 664 & 491.4146 & -0.476 \\
\hline 447 & 478.8253 & 0.179 & 501 & 481.9544 & -0.048 & 569 & 485.9045 & -0.166 & 665 & 491.4719 & -0.493 \\
\hline 447 & 478.8274 & 0.215 & 504 & 482.1375 & 0.098 & 584 & 486.7694 & -0.303 & 666 & 491.5400 & -0.322 \\
\hline 457 & 479.4087 & 0.204 & 505 & 482.1974 & 0.127 & 585 & 486.8333 & -0.206 & 668 & 491.6425 & -0.560 \\
\hline 458 & 479.4702 & 0.262 & 509 & 482.4295 & 0.116 & 586 & 486.8901 & -0.229 & 681 & 492.3960 & -0.611 \\
\hline 460 & 479.5823 & 0.188 & 510 & 482.4819 & 0.017 & 587 & 486.9451 & -0.285 & 682 & 492.4595 & -0.520 \\
\hline 461 & 479.6400 & 0.180 & 511 & 482.5406 & 0.025 & 600 & 487.6953 & -0.392 & 699 & 493.4440 & -0.602 \\
\hline 464 & 479.8154 & 0.194 & 512 & 482.6006 & 0.056 & 601 & 487.7651 & -0.193 & 705 & 493.7895 & -0.665 \\
\hline 465 & 479.8748 & 0.214 & 515 & 482.7719 & -0.000 & 601 & 487.7622 & -0.243 & 706 & 493.8516 & -0.597 \\
\hline 466 & 479.9279 & 0.127 & 516 & 482.8296 & -0.008 & 603 & 487.8830 & -0.167 & 707 & 493.9032 & -0.711 \\
\hline 470 & 480.1643 & 0.188 & 516 & 482.8326 & 0.042 & 604 & 487.9327 & -0.312 & 722 & 494.7758 & -0.715 \\
\hline 471 & 480.2222 & 0.185 & 517 & 482.8892 & 0.015 & 612 & 488.3968 & -0.337 & 723 & 494.8371 & -0.662 \\
\hline 472 & 480.2755 & 0.100 & 518 & 482.9433 & -0.054 & 613 & 488.4523 & -0.383 & 724 & 494.8931 & -0.700 \\
\hline 474 & 480.3975 & 0.197 & 526 & 483.4092 & -0.047 & 614 & 488.5193 & -0.231 & 733 & 495.4140 & -0.747 \\
\hline 475 & 480.4534 & 0.157 & 527 & 483.4678 & -0.040 & 615 & 488.5730 & -0.308 & 739 & 495.7585 & -0.828 \\
\hline 476 & 480.5116 & 0.157 & 528 & 483.5244 & -0.068 & 618 & 488.7454 & -0.347 & 740 & 495.8167 & -0.828 \\
\hline 477 & 480.5736 & 0.224 & 529 & 483.5860 & -0.010 & 619 & 488.7985 & -0.434 & 785 & 498.4271 & -0.968 \\
\hline 478 & 480.6261 & 0.125 & 532 & 483.7593 & -0.032 & 620 & 488.8621 & -0.341 & 786 & 498.4876 & -0.929 \\
\hline 480 & 480.7420 & 0.117 & 533 & 483.8188 & -0.010 & 621 & 488.9207 & -0.333 & & & \\
\hline 481 & 480.7976 & 0.072 & 534 & 483.8748 & -0.047 & 630 & 489.4422 & -0.372 & & & \\
\hline
\end{tabular}

${ }^{a}$ Individual errors in these timings are not explicitly included here.

${ }^{\mathrm{b}} \mathrm{E}$ (cycle number).

${ }^{\mathrm{c}}$ Superhump maxima expressed as HJD - 2,456,000.

${ }^{\mathrm{d}} \mathrm{O}-\mathrm{C}$ value (in cycles) according to the ephemeris.

$\mathrm{T}_{\text {_max }}(\mathrm{HJD})=2,456,452.8035+0.058191 \mathrm{E}$.

The $\mathrm{O}-\mathrm{C}$ residuals of the times of maximum light relative to the ephemeris given by equation 1 are shown in the lower panel of Figure 1. The resulting $\mathrm{O}-\mathrm{C}$ diagram is complex, but it displays a number of features that are usually observed in other SU UMa-type systems (Kato 2015). Among the most relevant features visible in this diagram we point out the following:

1. During the first four days of the outburst a weak modulation (early superhumps) with a period $P_{\text {esh }} \approx 0.057 \mathrm{~d}$ was visible in the light curve.

2. The onset of fully-grown (Stage $A$ superhumps) took place in a short time-scale $(\approx 2 \mathrm{~d})$ and involved an increase in the amplitude of the modulations. Their (mean) period, $P_{\operatorname{sh}(\mathrm{A})} \approx 0.059 \mathrm{~d}$, was longer than $P_{\text {esh }}$.
3. Once the superhump modulation reached full amplitude, the system entered Stage $B$ where the amplitude of the superhump decreased slowly, and the mean period became shorter $\left(P_{\mathrm{sh}(\mathrm{B})} \approx 0.058 \mathrm{~d}\right)$. The upward curvature of the residuals during this stage (days HJD 449-466) signifies that the period of the superhumps was not constant, but increased over time. From a quadratic fit of the residuals in this interval, we find an increase rate of $d P_{\mathrm{sh}(\mathrm{B})} / d t=5.8(4) \times 10^{-5}$.

4. Before the end of the main eruption, the amplitude of the superhumps was found to grow larger $(\approx 0.10 \mathrm{mag})$. The system entered Stage $C$, extending from day HJD 466 to the end of the main plateau (around day HJD 470), where the period of the superhump remained essentially constant $\left(P_{\mathrm{sh}(\mathrm{C})} \approx 0.0582 \mathrm{~d}\right)$. 

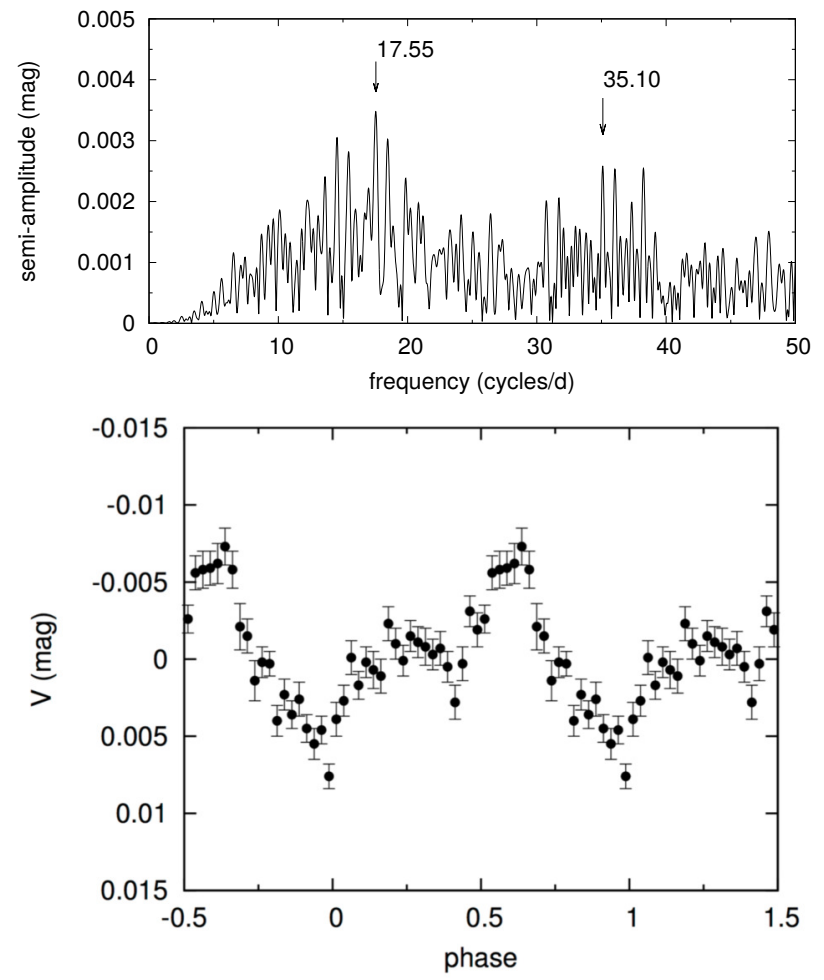

Fig. 2. Upper frame: Power spectrum during days HJD 445-448 (early superhumps), showing broad peaks centred at 17.55 cycles $\mathrm{d}^{-1}$ and its first harmonic. Lower frame: Waveform of the early superhumps obtained after folding the data with $P_{\text {esh }}=0.05698 \mathrm{~d}$. The zero phase is arbitrary.

5. Worth noting is the increase of the amplitude variations as the system dropped by about 3 mag between Stage $C$ and the post-outburst stage.

6. After the end of the main eruption (day $\geq$ HJD 473) the superhumps were still visible with a significantly larger amplitude $\approx 0.2 \mathrm{mag}$ ), and with a period which remained constant for at least the subsequent $\approx 25$ days. The period of the post-outburst modulation was shorter than $P_{\text {sh }}$.

A summary of the main periodicities along the eruption is given in Table 2, as found in the next subsections.

\subsubsection{Early Superhumps}

From the beginning of our campaign, a weak modulation of about 0.010 mag full amplitude was observed in the light curves. This signal persisted over days HJD 445-448. The power spectrum of the spliced light curve covering this 4-day segment is shown in the upper frame of Figure 2. It is dominated by two broad peaks centred at frequencies $35.10(3)$ and $17.55(3)$ cycles $\mathrm{d}^{-1}$. These signals were weak, with amplitudes of 0.0036 and $0.0030 \mathrm{mag}$, respectively. Although they were barely detected above the noise, we interpret them as a likely manifestation of early superhumps (Kato et al. 2014).

This photometric feature is known to be typical of WZ Sge-type stars, and is not shown by any other type of dwarf nova. Although its physical origin is still under debate, there is increasing observational evidence that its period $\left(P_{\text {esh }}\right)$ is essentially equal to $P_{\text {orb }}$ (Patterson et al. 1996; Kato 2015). A folded curve of the spliced light curve with $P_{\text {esh }}=0.05698(9) \mathrm{d}$ is also shown in the lower frame of Figure 2, which shows the double-humped pattern characteristic of early superhumps. The value of $P_{\text {esh }}$ obtained in this paper is slightly different from, but consistent with, the value of $0.05706(2) \mathrm{d}$ reported in Kato et al. (2014).

\subsubsection{Common Superhumps}

The double-humped pattern of the early superhumps turned into single-peaked humps on day HJD 449. Over days HJD 449-451, the mean amplitude was around $\approx 0.007$ mag and the period was $\approx 4 \%$ longer than the period found for early superhumps. The dominant signal occurred at 16.83(8) cycles $\mathrm{d}^{-1}$ corresponding to a period of $0.0594(3) \mathrm{d}$. Since the modulation was better defined in this 2-day interval, we were able to determine the times of maximum in the signal. We identified a total of 9 maxima in the HJD 449.6-451.6 day interval, and obtained a period of $0.05934(11) \mathrm{d}$ (corresponding to a frequency of $16.85(3)$ cycles $\mathrm{d}^{-1}$ ) from a linear regression. This value is fully consistent with the one found from the Fourier analysis. The modulation over this 2 -day segment is interpreted as Stage- $A$ superhumps. The period we find is close to, but slightly different from, the value of 0.05883(6) d reported in Kato et al. (2014).

Fully-grown, large-amplitude superhumps were finally observed on day HJD 452 (amplitude of $0.10 \mathrm{mag}$ ). As a representative example, we show in the upper frame of Figure 3 the light curve from day HJD 455. As the eruption proceeded, the mean amplitude of the superhumps decreased (as shown in the middle panel of Figure 3). The variation in amplitude was smooth in the HJD 452-466 day interval. We formed a spliced light curve in this interval, and obtained the power spectrum shown in the middle frame of Figure 3. The strongest signals occurred at $f_{1}=17.128(3)$ and $f_{2}=34.265(3)$ cycles $\mathrm{d}^{-1}$. 

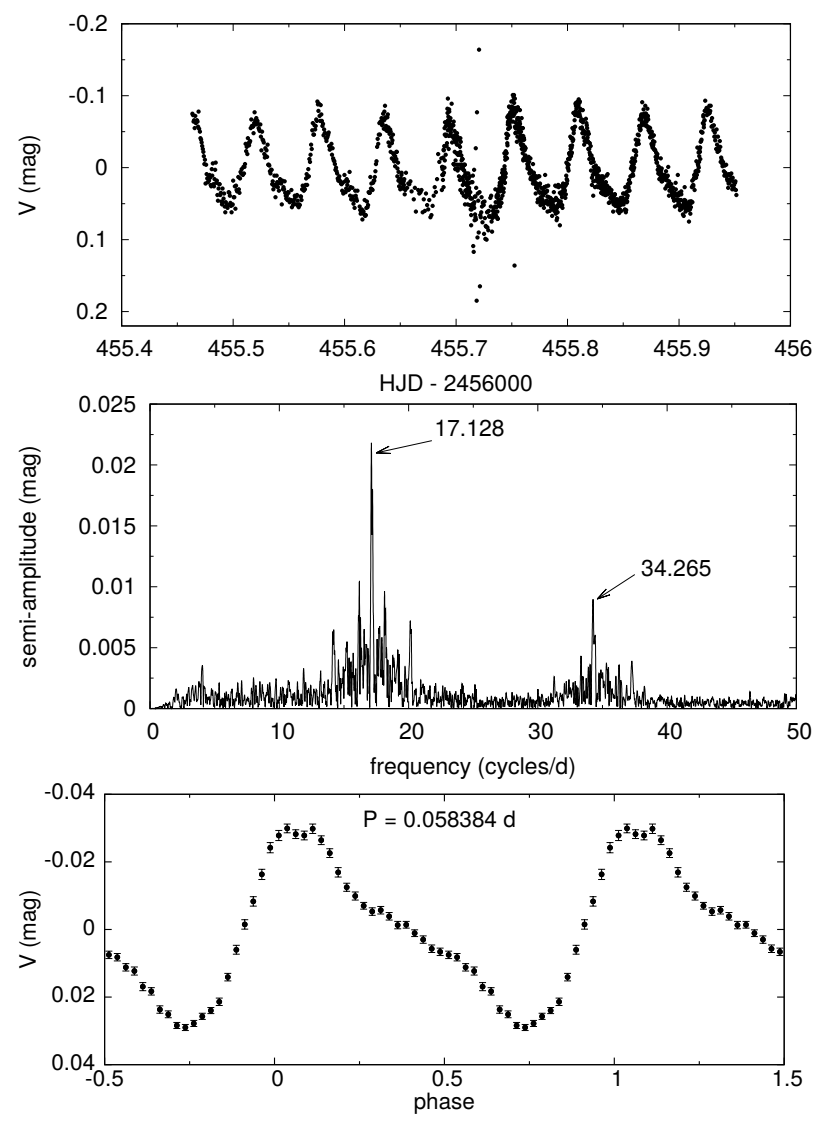

Fig. 3. Upper frame: A 12-hour spliced light curve obtained on day HJD 455, dominated by large-amplitude common superhumps. The zero level in the figure corresponds to $V \approx 12.1$ mag. Middle frame: Power spectrum during the common superhump era (days HJD 442466), with main peaks centred at frequencies 17.128 and 34.265 cycles $\mathrm{d}^{-1}$. Lower frame: Mean waveform of the common superhump obtained after folding the data on $P=0.058384 \mathrm{~d}$. The zero phase is arbitrary.

They were interpreted as the frequency of Stage- $B$ superhumps (period of $P_{\mathrm{sh}}=0.058384(10) \mathrm{d}$ ) and its first harmonic, respectively. Other (weaker) peaks, not shown in Figure 3, were found at $f_{3}=51.381(6)$ and $f_{4}=68.403(6)$ cycles $^{-1}$. The mean waveform of the superhump modulation during this interval is shown in the lower panel of Figure 3.

We note that after subtracting the superhump signal and its harmonics, the power spectrum of the residual light curve showed peaks at 17.20 and 17.28 cycles $\mathrm{d}^{-1}$. But we do not give any physical significance to these detections, and interpret them as the result of period and amplitude variations of the superhump wave during the eruption.

The amplitude of the superhumps increased around day HJD 466, and decreased thereafter until the end of the main eruption (Stage $C$ ). The
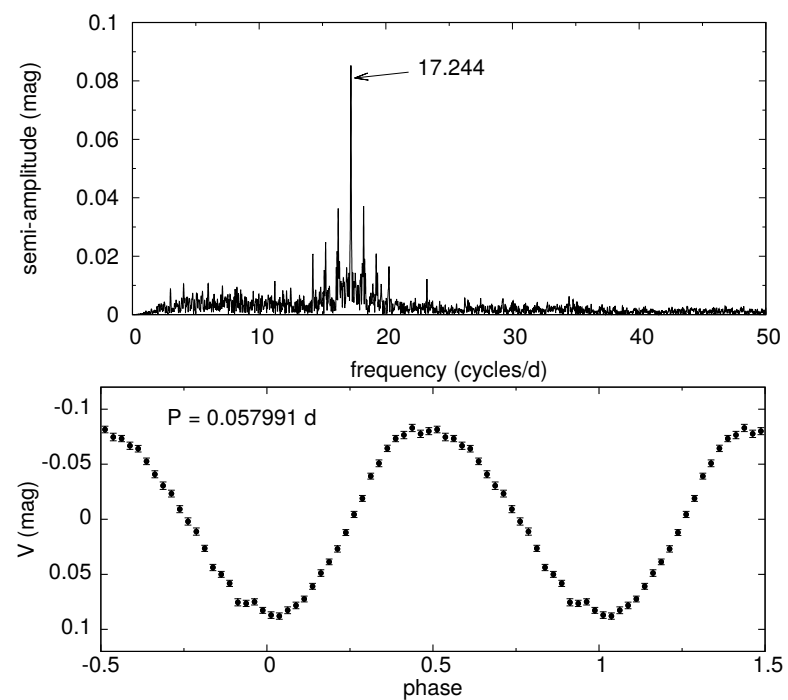

Fig. 4. Upper frame: Power spectrum after the main eruption (day HJD $\geq 473$ ) showing a strong peak centred at 17.244(1) cycles $\mathrm{d}^{-1}$ (post-outburst superhump). Lower frame: Mean waveform of the post-outburst superhump, obtained after folding the data on $P=$ $0.057991 \mathrm{~d}$. The zero phase is arbitrary.

strongest signal in the power spectrum in the HJD 466-470 day interval occurred at 17.191(10) cycles $\mathrm{d}^{-1}$, corresponding to a period of $0.05817(3) \mathrm{d}$, with additional peaks at higher harmonics.

\subsubsection{Post-Outburst Stage}

Once the main eruption was over, the light curve was still dominated by superhumps, but now with significantly larger amplitude $(\approx 0.15 \mathrm{mag})$ which decreased slowly $\left(\approx 0.013 \mathrm{mag} \mathrm{d}^{-1}\right)$. This behaviour remained essentially unchanged for nearly 20 days of our observations after the main fading.

We formed a spliced light curve including all the observations from day HJD $\geq 473$, and found a power spectrum (shown in the upper frame of Figure 4), with a peak at 17.244(1) cycles $\mathrm{d}^{-1}$. This was interpreted as the frequency of the post-outburst superhump, and dominated the spectrum. Higherorder harmonics were also found, but their amplitude was very low $(<0.0065 \mathrm{mag})$. This signifies that the waveform of the post-outburst superhump was nearly sinusoidal. The lower frame of Figure 4 shows that this was indeed the case.

\subsection{Photometry Near and at Minimum Light}

As detailed in $\S 2$, we took two runs near minimum light covering around one orbital period each. The light curves are shown in the upper panel of Figure 5. When folded with the orbital period 
TABLE 2

\section{MEAN PHOTOMETRIC PERIODS AND FREQUENCIES OF THE SUPERHUMP MODULATION.*}

\begin{tabular}{cllc}
\hline $\begin{array}{c}\text { Time interval } \\
\text { (HJD-2456000) }\end{array}$ & \multicolumn{1}{c}{$\begin{array}{c}\text { Period } \\
(\mathrm{d})\end{array}$} & $\begin{array}{c}\text { Frequency } \\
\left(\text { cycles d }^{-1}\right)\end{array}$ & Comments \\
\hline $445-448$ & $0.05698(9)$ & $17.55(3)$ & early superhumps \\
$449-451$ & $0.0594(3)$ & $16.83(8)$ & Stage $A$ \\
$452-466$ & $0.058384(10)$ & $17.128(3)$ & Stage $B$ \\
$466-470$ & $0.05817(3)$ & $17.191(10)$ & Stage $C$ \\
$473-498$ & $0.05799(1)$ & $17.244(1)$ & post outburst \\
$449-498$ & $0.0581910(1)$ & $17.1847(3)$ & mean superhump period \\
\hline
\end{tabular}

*Values correspond to the 2013 superoutburst of V1838 Aql. The errors in parentheses correspond to the last significant figures.

$(0.05698 \mathrm{~d})$, the light curves seem to be out of phase. But this is not surprising: over the 23 days (nearly 400 orbital cycles) elapsed between both runs, an uncertainty of 0.0001 days in $P_{\text {orb }}$ involves an uncertainty of 0.7 in phase. We carried out a period analysis of both nights using the Phase Dispersion Minimization (PDM) technique (Stellingwerf 1978) in the Peranso package (Paunzen \& Vanmunster 2016). This technique is frequently used to detect variations of superhumps in SU UMa systems (e.g. Kato et al. 2014). The lower frame in Figure 5 shows the results of combining the two nights with the best period estimate $(0.0576 \mathrm{~d})$ determined from the PDM technique. Assuming that the observed light comes from the accretion disc, the zero point obtained in this case is HJD 2456537.6946 (time of inferior conjunction of the secondary). The period found using the PDM method yielded a value which is still close to the post-outburst state, but the sinusoidal shape is gone. There was only a small peak around phase 0.25 . No double modulation with orbital period was found as would be expected in a bounce-back object. Further observations in the $R$ band were obtained on 2018, July 18 covering three orbital cycles. Since the night was not photometric, we were unable to make absolute calibrations and only differential photometry is shown in Figure 6. No obvious orbital modulation was detected within the individual errors, which are rather large $(\approx 0.03 \mathrm{mag})$.

\section{DISCUSSION}

The values derived for the superhump period in Stages $A$ and $B$ allowed us to estimate the mass ratio of the system through the known "superhump excess" $\epsilon-q$ relations (Patterson 1998, 2011; Kato \& Osaki 2013). Considering the lack of a reliable determination of the orbital period from spectroscopic obser-

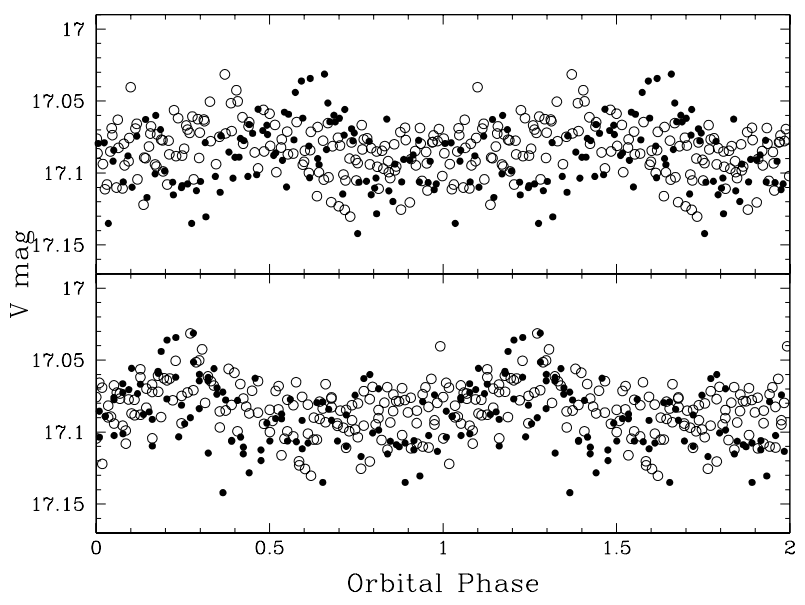

Fig. 5. Upper panel: $V$ light curves obtained near minimum light in 2013. Open dots are from September 2 and filled dots from September 25. The size of the points corresponds to the mean individual errors. The orbital phases have been taken from the ephemeris obtained in this paper. Lower panel: The two nights folded with the ephemeris obtained by using the PDM technique (see more details in text).

vations, we assume here that $P_{\text {orb }}$ is equal to the period of early superhumps. We find $\epsilon_{A}=0.042(5)$ and $\epsilon_{B}=0.024(2)$. Thus, our estimates for the mass ratio are $q_{A}=0.12(2)$ and $q_{B}=0.10(1)$, respectively. Comparing these two values with the $\epsilon-q$ relation shown in Bakowska et al. (2017, Figure 19 ) we can see clearly that $\epsilon_{B}$ is well within the expected value, while $\epsilon_{A}$ is not. This is further supported by using the updated Stolz \& Schoembs (1984) relation in Otulakowska-Hypka et al. (2016, equation 4), which for our assumed orbital period gives $\epsilon=0.019(10)$. Although we are inclined to use the Stage $B$ results, we point out that both values are suggestive of a 


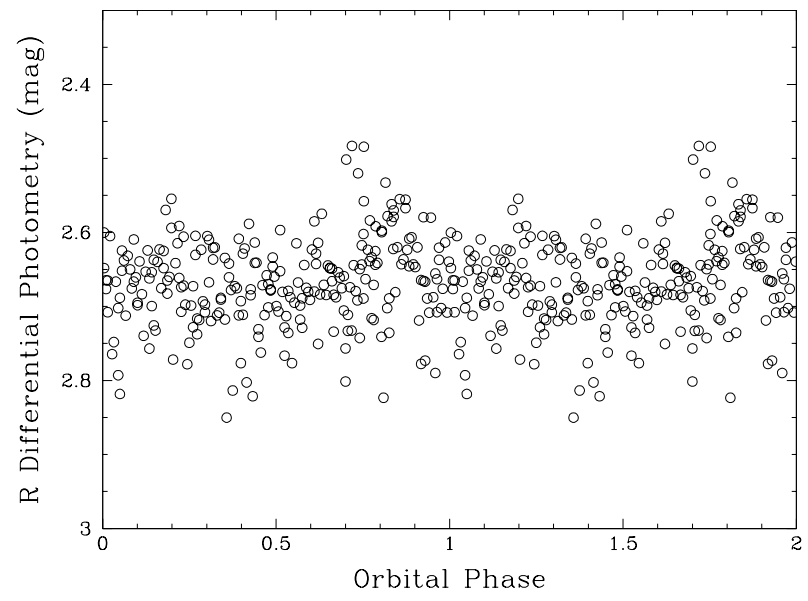

Fig. 6. Differential $R$ light curve obtained at minimum light in July 18, 2018. The size of the individual errors $(\approx 0.03 \mathrm{mag})$ is rather large. The orbital phases have been taken from the ephemeris obtained in this paper. No obvious orbital modulation, within the errors, is detected at this level.

low-mass donor, although as pointed out in $\S 1$ (e.g. Otulakowska-Hypka et al. 2016, see their Figure 7), empirical relations $\epsilon-q$ at low $q$ values may carry large systematic uncertainties. For a typical white dwarf with mass $\approx 0.8 M_{\odot}$ (Zorotovic, Schreiber \& Gänsicke 2011) and mass ratio $q \approx 0.1$, the mass of the secondary is very close to the substellar limit i.e. $0.072 M_{\odot}$ (Chabrier \& Baraffe 2000). Further characterisation of systems like V1838 Aql will allow us to discern empirically where this limit lies for masslosing donors.

It has been noted by many authors, both theoretically and observationally, that the CV orbital period distribution should present a sharp cut-off at about $\approx 80 \mathrm{~min}$, usually termed as the minimum period (e.g. Rappaport et al. 1982; Ritter \& Kolb 1998; Gänsicke et al. 2009). V1838 Aql has an orbital period of about $82 \mathrm{~min}$, very close to the minimum period, which makes it difficult to discern whether it is approaching to, or receding from, this minimum orbital period. Before asserting its true nature, we could look at some observational features in those CVs systems around the minimum orbital period. Most of these systems possess WZ Sge-like features. Their optical spectra are mostly dominated by the white dwarf and accretion disc itself, with no visible features from the donor. Since the rate of accretion is an order of magnitude smaller than that for systems before reaching the minimum pe$\operatorname{riod}\left(\dot{m} \approx 10^{-11} M_{\odot} \mathrm{yr}^{-1}\right)$, the accretion discs become very faint, and the broad absorption lines of the white dwarf become visible below $\approx 5000 \AA$ ) e.g.
WZ Sge (Howell et al. 2008). However, the donor's observed properties should vary significantly for systems with the same orbital period but evolving towards or away from the period minimum. This is a consequence of the donor's temperature steep relationship as a function of orbital period (e.g. Knigge et al. 2011). From the superhump analysis presented here, we suspect that the system is approaching the period minimum and thus, the donor is probably a late-M dwarf with an observed effective temperature of $\approx 2400 \mathrm{~K}$ (Knigge et al. 2011).

The donor of V1838 Aql is therefore an ideal candidate for NIR time-resolved spectroscopy (e.g. SDSS J143317.78+101123.3, Hernández Santisteban et al. 2016), which would render a fully independent measurement of the orbital period and the mass ratio, to confirm or reject its substellar nature. This is particularly important since few low-q systems have been observed in outburst and for which a dynamical measurement of their components is feasible. (Figure 3 in Kato \& Osaki 2013). Thus, V1838 Aql could be a system to calibrate the empirical superhump relations in this poorly-explored region of parameter space.

\section{CONCLUSIONS}

We have presented a long-term study of the 2013 superoutburst of V1838 Aql from its peak to quiescence. Our main results are as follows:

- The observed early superhumps suggest an orbital period of $P_{\text {orb }}=0.05698(9)$ d, which locates V1838 Aql close to the minimum of the orbital period distribution in CVs.

- From Stages $A$ and $B$ and early superhump periods, we found the mass ratio to be $q_{A}=0.12(2)$ and $q_{B}=0.10(1)$, respectively.

- Based on the obtained values of the mass ratio, we claim that the donor in V1838 Aql is a low-mass star rather than a substellar object, and that the system is approaching the period minimum.

Given the long interval between outbursts in low $q$ systems, it is of paramount importance to confirm by dynamical methods the orbital parameters of such systems. This would indicate which systems may be used in order to calibrate the empirical superhump excess relations.

The authors are indebted to DGAPA (Universidad Nacional Autónoma de México) support, 
PAPIIT projects IN111713, IN122409, IN100617, IN102517, IN102617, IN108316 and IN114917. GT acknowledges CONACyT grant 166376. JE acknowledges support from a LKBF travel grant to visit the API at UvA. JVHS is supported by a Vidi grant awarded to N. Degenaar by the Netherlands Organization for Scientific Research (NWO) and acknowledges travel support from DGAPA/UNAM. E. de la F. wishes to thank CGCI-UdeG staff for mobility support. We thank the day and night-time support staff at the OAN-SPM for facilitating and helping obtain our observations.

\section{REFERENCES}

Bąkowska, K., Olech, A., Pospieszyński, R., et al. 2017, A\&A, 603, 72

Breedt, E., Gänsicke, B. T., Drake, A. J., et al. 2014, MNRAS, 443, 3174

Chabrier, G. \& Baraffe, I. 2000, ARA\&A, 38, 337

Coppejans, D. L., Körding, E. G., Knigge, C., et al. 2016, MNRAS, 456, 4441

de Miguel, E., Patterson, J., Cejudo, D., et al. 2016, MNRAS, 457, 1447

Gänsicke, B. T., Dillon, M., Southworth, J., et al. 2009, MNRAS, 397, 2170

Harrison, T. E. 2016, ApJ, 816, 4

Hernández Santisteban, J. V., Knigge, C., Littlefair, S. P., et al. 2016, Natur, 533, 366

Howell, S. B., Rappaport, S., \& Politano, M. 1997, MNRAS, 287, 929

Howell, S. B., Hoard, D. W., Brinkworth, C., et al. 2008, ApJ, 685, 418

Itagaki, K. et al. 2013, CBET, 3554, 1

Kato, T. 2015, PASJ, 67, 108

Kato, T., et al. 2009, PASJ, 61, 395
Kato, T. et al. 2014, PASJ, 66, 90

Kato, T. \& Osaki, Y. 2013, PASJ, 65, 115

Knigge, C., Baraffe, I., \& Patterson, J. 2011, ApJS, 194, 28

Lenz P., \& Breger M. 2005, CoAst, 146, 53

Littlefair, S. P., Dhillon, V. S. \& Martin, E. L. 2003, MNRAS, 340, 264

Littlefair, S. P., Dhillon, V. S., Marsh, T. R., et al. 2006, Sci, 314,1578

Neustroev, V. V., Marsh, T. R., Zharikov, S. V., et al. 2017, MNRAS, 467, 597

O'Donoghue, D., Chen, A., Marang, F., Mittaz, J. P. D., Winkler, H. \& Warner, B. 1991, MNRAS, 250, 363

Osaki, Y. 1974, PASJ, 26, 429 1994, ASIC, 417, 93

Otulakowska-Hypka, M., Olech, A., \& Patterson, J. 2016, MNRAS, 460, 2526

Paczynski, B. \& Sienkiewicz, R. 1981, ApJ, 248, 27

Patterson, J. 1998, PASP, 110, 1132 2011, MNRAS, 411, 2695

Patterson, J. et al. 1996, PASP, 108, 748

Patterson, J. et al. 2005, PASP, 117, 1204

Paunzen, E. \& Vanmunster, T. 2016, AN, 337, 239

Rappaport, S., Joss, P. C., \& Webbink, R. F. 1982, ApJ, 254,616

Ritter, H. \& Kolb, U. 1998, A\&A, 129, 83

Skillman D. R. \& Patterson J. 1993, ApJ, 417, 298

Smak, J. 1993, AcA, 43, 101

Stellingwerf, R. F. 1978, ApJ, 224, 953

Stolz, B. \& Schoembs, R. 1984, A\&A 132, 187

Vogt, N. 1982, ApJ, 252, 653

Warner, B. 1995, Cataclysmic Variable Stars (Cambridge, MA: CUP)

Whitehurst, R. 1988, MNRAS, 232, 35

Zorotovic, M., Schreiber, M. R., \& Gänsicke, B. T., 2011, A\&A, 536, A42

G. Bolt: CBA-Australia, 295 Camberwarra Drive, Craigie, Western Australia 6025, Australia.

R. Costero, C. Echevarría, A. Ruelas-Mayorga, and L. J. Sánchez: Instituto de Astronomía, Apartado Postal 70-264, México, CDMX, C.P. 04510 (jer@astro.unam.mx).

E. de Miguel: Departamento de Ciencias Integradas, Universidad de Huelva, E-21071 Huelva, Spain.

W. Goff: CBA-California, 13508 Monitor Lane, Sutter Creek, CA 95685, USA.

D. González-Buitrago: Departament of Physics and Astronomy 4129, Frederick Reines Hall, University of California, Irvine, CA 92697-4575, USA.

F.-J Hambsch: CBA-Mol, Andromeda Observatory, Oude Bleken 12, B-2400 Mol, Belgium.

J. V. Hernández Santisteban: Anton Pannekoek Institute for Astronomy, University of Amsterdam, Science Park 904, 1098XH Amsterdam, The Netherlands.

J. L. Jones: CBA-Oregon, Jack Jones Observatory, 22665 Bents Road NE, Aurora, OR, USA.

R. Michel: Instituto de Astronomía, Universidad Nacional Autónoma de México, Apartado Postal 877, Ensenada, Baja California, C.P. 22830, México.

J. Olivares: Laboratoire d'astrophysique de Bordeaux, Univ. Bordeaux, CNRS, B18N, allée Geoffroy Saint-Hilaire, 33615 Pessac, France.

A. Oskanen: CBA-Finland, Hankasalmi Observatory, Verkkoniementie 30, FI-40950 Muurame, Finland.

R. Sabo: CBA-Montana, 2336 Tailcrest Dr., Bozeman, MT 59718, USA.

D. Slauson: CBA-Iowa, Owl Ridge Observatory, 73 Summit Avenue NE, Swisher, IA 52338, USA.

W. Stein: CBA-Las Cruces, 6025 Calle Paraiso, Las Cruces, NM 88012, USA.

J. Ulowetz: CBA-Illinois, Northbrook Meadow Observatory, 855 Fair Lane, Northbrook, IL 60062, USA. 